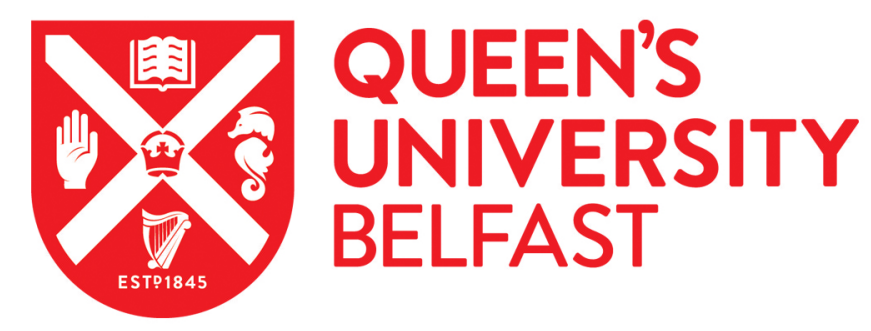

\title{
Single-cell RNA sequencing study of retinal immune regulators identified CD47 and CD59a expression in photoreceptors - implications in subretinal immune regulation
}

Liu, J., Tang, M., Harkin, K., Du, X., Luo, C., Chen, M., \& Xu, H. (2020). Single-cell RNA sequencing study of retinal immune regulators identified CD47 and CD59a expression in photoreceptors - implications in subretinal immune regulation. Journal of Neuroscience Research, 98(7), 1458-1513. https://doi.org/10.1002/jnr.24618

\section{Published in:}

Journal of Neuroscience Research

\section{Document Version:}

Peer reviewed version

Queen's University Belfast - Research Portal:

Link to publication record in Queen's University Belfast Research Portal

\section{Publisher rights}

(c) 2020 Wiley Periodicals, Inc. This work is made available online in accordance with the publisher's policies. Please refer to any applicable terms of use of the publisher.

\section{General rights}

Copyright for the publications made accessible via the Queen's University Belfast Research Portal is retained by the author(s) and / or other copyright owners and it is a condition of accessing these publications that users recognise and abide by the legal requirements associated with these rights.

Take down policy

The Research Portal is Queen's institutional repository that provides access to Queen's research output. Every effort has been made to ensure that content in the Research Portal does not infringe any person's rights, or applicable UK laws. If you discover content in the

Research Portal that you believe breaches copyright or violates any law, please contact openaccess@qub.ac.uk. 


\section{Single-cell RNA sequencing study of retinal immune regulators identified CD47 and CD59a expression in photoreceptors - implications in subretinal immune regulation}

Running title: Immune regulation in the retina

Jian Liu", \# Miao Tang ${ }^{2}$,\#, Kevin Harkin ${ }^{2}$, Xuan $\mathrm{Du}^{2}$, Chang Luo ${ }^{1}$, Mei Chen ${ }^{2}$, Heping Xu ${ }^{1,2, *}$

${ }^{1}$, Aier Eye Institute, Aier School of Ophthalmology, Central South University, P.R. China.

${ }^{2}$, The Wellcome-Wolfson Institute for Experimental Medicine, School of Medicine, Dentistry and Biomedical Sciences, Queen's University Belfast, UK.

\#, The authors contributed equally to this work.

*, Correspondence to: Heping $\mathrm{Xu}$, The Wellcome-Wolfson Institute for Experimental Medicine, School of Medicine, Dentistry and Biomedical Sciences, Queen's University Belfast, 97 Lisburn Road, Belfast, BT9 7BL, UK. Tel: 44(0)28909 76463; Email: heping.xu@ qub.ac.uk 


\section{Significance}

Retinal neurons sense light and give rise vision, whereas microglia protect neurons from eternal and external insults and maintain homeostasis. Uncontrolled microglial activation can damage neurons and impair vision. We show that different retinal neurons express different types of immune inhibitors. The light-sensing photoreceptors, in particular, express CD47 and CD59a, which are known to have strong immune suppressive functions. The expression of CD47 and CD59a is upregulated in the diseased eyes. Importantly, we found that photoreceptors can enhance anti-inflammatory and reduce pro-inflammatory gene expression in microglia. Our results highlight the role of light-sensing neurons in regulating retinal neuroinflammation. 


\begin{abstract}
The neuroretina is protected by its own defence system i.e., microglia and the complement system. Under normal physiological conditions, microglial activation is tightly regulated by neurons although the underlying mechanism remains elusive. Using published single-cell RNA sequencing datasets, we found that immune regulatory molecules including CD200, CD47, CX3CL1, TGF $\beta$ and complement inhibitor CD59a are expressed by various retinal neurons. Importantly, we found that photoreceptors express higher levels of CD47 and CD59a, which was further confirmed in cultured 661W cells, WERI-Rb1 cells and microdissected photoreceptors from human eyes. The expression of CD59a mRNA in 661W cells was upregulated by $\mathrm{TNF} \alpha$ and hypoxia. Whereas, LPS, hypoxia and IL-4 upregulated CD47 mRNA expression in 661W cells. Immunofluorescence staining detected strong CD59a immunoreactivity in the outer nuclear layer, inner/outer segments and discrete staining in ganglion cell layer (GCL), inner plexiform layer (IPL) and outer plexiform layer. The expression of CD59a in photoreceptors was increased in the detached retina, but decreased in retinas from experimental autoimmune uveoretinitis (EAU) mice. In EAU retina, CD59a is highly expressed by active immune cells. CD47 was detected in GCL, IPL and inner nuclear layer (INL) and some photoreceptors. The expression of CD47 in photoreceptors is also increased in the detached retina but decreased in EAU retina. In a co-culture system, 661W enhanced arginase- 1 and reduced IL-6 mRNA expression in BV2 microglial cells. Our results suggest that photoreceptors express immune regulatory molecules and may have the potential to regulate immune activation in the outer retina/subretinal space under pathophysiological conditions.
\end{abstract}

Key words: retina, neurons, photoreceptor, microglia, complement system, immune regulation 


\section{Introduction}

The neuroretina utilizes physical barriers (i.e., the inner blood retina barrier-iBRB, the outer blood retina barrier - oBRB) to avoid external insults and the immune suppressive microenvironment to reduce the negative impact caused by pathogenic response(Taylor \& $\mathrm{Ng} 2018$ ). The avoidance and tolerance strategies ensure immune privilege (IP) of the neuroretina(Chen et al. 2019). The retina also has its own defence system executed predominately by microglia and the complement system (Chen et al. 2019). This immune defence system is operational when the retina suffers from endogenous insults such as oxidative damage during aging ( $\mathrm{Xu}$ et al. 2009) or when IP is breached (Chen \& Xu 2015). The level of immune response (i.e., inflammation) depends on the level of insults as well as regulatory signals in the retinal microenvironment. The loss of retinal IP and uncontrolled defence response may lead to sight-threatening retinopathy such as age-related macular degeneration (AMD), diabetic retinopathy (DR) and uveoretinitis (Forrester \& Xu 2012)

The neuron-microglia interactions are important for the central nervous system (CNS) homeostasis (Szepesi et al. 2018). Microglia are the principle cells responsible for synaptic pruning (Schafer et al. 2013) and clearance of neuronal debris (Arcuri et al. 2017). Neurons, on the other hand, send signals to control microglial activation (Szepesi et al. 2018). For example, CX3CL1 (fractalkine), TGF $\beta$ and CD200 produced by healthy neurons can inhibit microglial activation (Szepesi et al. 2018). Retinal microglia are located in the ganglion cell layer (GCL), inner plexiform layer (IPL) and outer plexiform layer (OPL)(Karlstetter et al. 2015, Chen \& Xu 2015). This distribution is in line with their homeostatic function in the retina, i.e., synaptic pruning and clearance of neuronal debris. A recent study has shown that retina has two microglia pools differing by their IL-34 dependency (O'Koren et al. 2019). The IL-34-dependent microglia in the IPL contribute to retinal neuronal functions under homeostatic conditions (O'Koren et al. 2019). The precise mechanism of neuron-microglia interactions in different retinal layers remains to be fully elucidated. 
The complement system is another important arm of retinal innate immune defence and is actively involved in neuron-microglia interactions (Xu \& Chen 2016). For example, the complement component C1q is necessary for synaptic pruning in the retina and CNS (Chu et al. 2010). The expression of $\mathrm{Clq}$ is upregulated and synaptically re-located in the early stages of glaucoma (Howell et al. 2011). Complement activation in the retina is tightly controlled and dysregulated complement activation is known to contribute to various sight-threatening conditions, including

DR, AMD and uveoretinitis (Xu \& Chen 2016). The role of retinal neurons in complement activation has not been fully investigated.

Although the general role of neurons in regulating retinal immune response is well-appreciated, the precise mechanism remains to be elucidated. The aim of this study was to understand the neuron-derived molecular cues that control retinal microglia and complement activation. In this regard, we examined the expression profile of various immune regulators by different retinal neurons. We found that retinal neurons including ganglion cells, amacrine cells, horizontal cells and photoreceptors (cone and rods) express various immune regulators. Importantly, we found that CD59a and CD47 are highly expressed in cones and rods.

\section{Materials and Methods}

\section{Single-cell RNA sequencing data processing}

Two retinal single-cell RNA sequencing (scRNA-seq) datasets (GSM3580725 and GSM3580727) (https://www.ncbi.nlm.nih.gov/) were downloaded from the GEO database. Samples in both GSM3580725 and GSM3580727 were obtained from 8 weeks C57BL/6J wild type male mice. Sample preparation and single cell sequencing were detailed in the authors' publication (Heng et al. 2019). Based on the information in the paper, libraries were sequenced using the Illumina 
HiSeq 2500 system and scRNA-seq data were pre-processed using the Cell Ranger pipeline (version 2.1.0; 10x Genomics) with default settings (Heng et al. 2019).

Two datasets were merged into one gene $\times$ cell counts matrix. The Seurat $\mathrm{R}$ package (version 3.0.0) was used to analyse the merged single-cell dataset (Butler et al. 2018). Common quality control criteria for scRNA-seq was calculated. Cells with 200 to 4500 genes and less than $20 \%$ of mitochondrial transcripts were retained for further analysis. Genes that were present in at least 10 cells in one sample were kept for subsequent analysis. A total of 14769 genes $\times 12769$ cells count matrix was obtained after quality control. Sctransform normalization, using regularized negative binomial regression, was performed. The SCTransform was used to normalize the dataset. The SCTransform solution adjusted the UMI counts using regularized negative binomial regression model, which would remove the variation due to sequencing depth. The Pearson residuals from the regularized negative binomial regression models were used as normalized UMI counts (Hafemeister C and Satija R. 2019). Furthermore, to eliminate unwanted cell-cell variation in gene expression, the percentage of mitochondrial gene expression was regressed out during the normalization. Principal components analysis (PCA) and Uniform Manifold Approximation and Projection (UMAP) were used for dimension reduction on normalized data matrix. All 50 PCs was kept and passed into UMAP. The cells were clustered in 32 groups and major retinal cell types were identified based on known markers (Table 1). 1793 out of the 12769 cells were excluded from subsequent analysis due to uncertain classification. No obvious batch effect was observed in these two datasets (Supplementary data 1). Log-normalization counts were used for data visualization.

\section{Animals}

C57BL/6J mice between 6-8 weeks of age (20-25g, both female and male) were provided by the Biological Service Unit of Queen's University Belfast. Animals were maintained in a Specificpathogen-free environment with controlled temperature $\left(22-23^{\circ} \mathrm{C}\right)$ on a $12 \mathrm{~h} \mathrm{light} / 12 \mathrm{~h}$ dark cycle. 
All mice had free access to food and water. At the end of the experiment, animals were humanely sacrificed by $\mathrm{CO} 2$ inhalation. Mouse eye sample collection was normally conducted in the afternoon. Five mice were used in each group, including (1) the expression of CD59 and CD47 in the normal retina, (2) retinal detachment (RD), (3) experimental autoimmune uveoretinitis (EAU), and (4) retinal explant cultures. Animals used to induce retinal detachment and EAU were randomized. Sample calculation, randomization and blinding were not performed. No exclusion criteria were pre-determined. The animal identification was not revealed to researcher who performed the immunofluorescence staining. All experimental protocols concerning animals in this study were performed according to the Association for Research in Vision and Ophthalmology (ARVO) Statement for the Use of Animals in Ophthalmic and Vision Research and approved by the Animal Welfare Ethical Review Body of Queen's University Belfast.

\section{Mouse models of retinal diseases}

Induction of RD: RD was induced in mice by subretinal injection of sodium hyaluronate detailed in our recent publication (Augustine et al. 2019). Mouse eyes were collected 7 days after induction of RD and processed for immunofluorescence staining.

Induction of EAU: EAU was induced in C57BL/6J mice by immunizing the animals with 500 mg of IRBP1-20 (GPTHLFQPSLVLDMAKVLLD, GL Biochem,Shanghai, China) using the protocol reported in our previous publications (Xu et al. 2008; Chen et al. 2018). Mouse eyes were collected 25 days after induction of EAU and processed for immunofluorescence staining.

\section{Human tissues}

Eyes from human donors were obtained from the Eye Bank of Aier Eye Hospital (Changsha, HN). The study protocol was in accordance with the Declaration of Helsinki and the principles set out in the Standard for Eye Bank issued by Ministry of Health of the People's Republic of China for research involving human tissues. Two pairs of eyes from donors aged 48 and 89 years 
respectively were used in this study. Both donors had no eye diseases and donor one (48 years old) died of accident and donor two (89 years old) died of old age. Eyes were collected within $8 \mathrm{~h}$ after death and fixed in Davidson's fixative solution $(110 \mathrm{ml} / \mathrm{L}$ Acetic acid; $320 \mathrm{ml} / \mathrm{L}$ of 99\% Ethanol; $222 \mathrm{ml} / \mathrm{L}$ of Formaldehyde-solution in 10\% phosphate buffered; $347 \mathrm{ml} / \mathrm{L}$ of deionized water) for $24 \mathrm{~h}$ before processing for histology and immunohistochemistry.

\section{Microdissection}

The eyes were embedded in optimal cutting temperature compound (OCT, Sakura, Cat\# 4583, Torrance, CA, USA) and cryosectioned (15 $\mu \mathrm{m}$ thickness). The collection of human photoreceptor component were performed under a fluorescence microscope using the methods described by Chan et al. (2005) with slight modification. The photoreceptors were visible under the fluorescence microscope due to the strong autofluorescent signals and the layers were gently scraped with a 26-gauge needle and immediately transfer to a RNA-free tube for total RNA isolation.

\section{Retinal explant culture}

The protocol of retinal explant culture was adapted from the protocol reported by Eandi et al. (2016). Briefly, the eyes were collected from 8-week old C57BL/6J female mice. The anterior segment (cornea, iris and ciliary body, and lens) of the eye was removed. The neural retina was carefully peeled off from the eyecup. The retinas were either immersed immediately in OCT compound and snap frozen (0h culture) or placed on top of a polycarbonate filter (Merck UK, Felthan, Cat\#PIHP01250) in DMEM/F12 + 10\% FCS $+1 \%$ penicillin and streptomycin (Thermo Fisher Scientific, Altrincham, UK, Cat\# 15140122) and cultured for 6 hours at $37^{\circ} \mathrm{C}$. The cultured explants were then immersed in OCT compound and processed further for immunostaining.

\section{Cell culture and treatments}


Mouse Photoreceptor cell line 661W culture: The 661W was generously provided by Dr. Muayyad Al-Ubaidi (Department of Cell Biology, University of Oklahoma Health Sciences Center, Oklahoma City, OK, USA) (the cell line is not listed as a commonly misidentified cell line by the International Cell Line Authentication Committee). The cells were grown in DMEM basic medium (Gibco, Shanghai, Cat\# C11885500BT ) supplemented with 10\% fetal calf serum (Gibco,

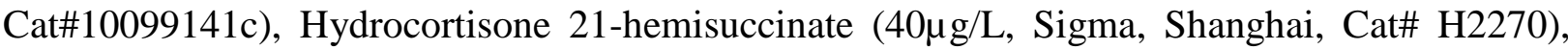

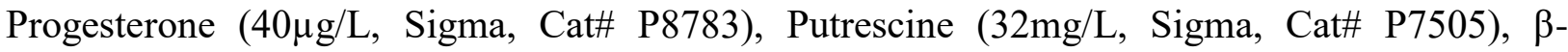
mercaptoethanol (0.04\%V/V, Life Technologies, Shanghai, Cat\# 21985-023), penicillin and streptomycin (Thermo Fisher Scientific, Cat\# 15140122) and cultured in $5 \% \mathrm{CO}_{2}$ at $37{ }^{\circ} \mathrm{C}$ incubator. Cells were not used for more than 10 passages.

Treatments: $661 \mathrm{~W}$ cells were seeded in 6 -well plates at a density of $2 \times 10^{5}$ cells/well. After reaching confluence, cells were treated with TNF- $\alpha$ (20ng/ml, R\&D Systems, Shanghai, Cat\# 410MT-025), lipopolysaccharide (LPS, 100 ng/ml, Sigma, Cat\# L3024), IL-4 (20 ng/ml, PeproTech, Suzhou, JS, Cat\# 214-14), hypoxia condition (5\% O 2 ), hydrogen peroxide (200 $\mu \mathrm{M}$, Sigma, Cat\#323381) for $18 \mathrm{~h}$ respectively. Cells were harvested for total RNA after $18 \mathrm{~h}$ and for protein after $48 \mathrm{~h}$. All the experiments were repeat at least 3 times.

WERI-Rb-1 cells culture: The WERI-Rb-1 cells were purchased from National Infrastructure of Cell Line Resource (NICR, Beijing, Cat\# 3131C0001001200012) (the cell line is not listed as a commonly misidentified cell line by the International Cell Line Authentication Committee). The cells were cultured in RPMI (Gibco, Cat\#RPM1-1640) containing 10\% Fetal Bovine Serum (FBS) and $1 \%$ penicillin-streptomycin at $37{ }^{\circ} \mathrm{C}$ in $5 \% \mathrm{CO}_{2}$ incubator. Cells were not used more than 10 passages.

BV2 microglial cells culture: The BV2 cells were purchased from National Infrastructure of Cell Line Resource (NICR, Beijing, Cat\# 3111C0001CCC000063) (the cell line is not listed as a 
commonly misidentified cell line by the International Cell Line Authentication Committee). The cells were cultured in DMEM (Gibco, Cat\#RPM1-1640) containing 10\% FBS and 1\% penicillinstreptomycin at $37^{\circ} \mathrm{C}$ in $5 \% \mathrm{CO} 2$ incubator. Cells were not used more than 10 passages.

Co-culture of BV2 and $661 \mathrm{~W}$ cells: BV-2 cells were cultured in 6-well plates at a density of $1.5 \times 10^{6}$ cells/well. The cells were then polarized to M1 (100 ng/ml LPS and $20 \mathrm{ng} / \mathrm{ml} \mathrm{IFN- \gamma}$ (PeproTech, Cat\# 315-05) or M2 (20 ng/ml IL-4) for $6 \mathrm{~h}$ or remain un-polarized (M0). The cells were washed twice with warm PBS and co-cultured 661W (BV2:661W = 5:1) for 24h. The cells were then detached in ice-cold PBS with $2 \mathrm{mM}$ EDTA and pass through $70 \mu \mathrm{m}$ cell strainer. BV2 cells were isolated using the CD11 $b^{+}$MACS kit (Miltenyi Biotec, China, Shanghai) and processed for further real-time RT-PCR analysis.

\section{Real-time qPCR}

Total RNA was extracted from the cells using the Total RNA Kit II (Omega, Norcross, GA, Cat\# R6934-01) according to the manufacturer's instructions. The quantity and quality of RNA was determined using a NanoDrop ND-1000 spectrophotometer (NanoDrop Technologies, Wilmington, DE). The same amount of total RNA was used to synthesise cDNA using the PrimeScript RT Reagent Kit (TaKaRa, Dalian, LN, Cat\# 6110A).

Real-time PCR assay was performed using SYBR Premix Ex Taq II (TaKaRa, Cat\#RR820Q) according to the manufacturer's instructions. The primers used in this study were designed using the NCBI Primer BLAST system and are listed in Table 2. GADPH was used as a housing-keeping gene. All real-time qPCR assays were determined using the LightCycler® 96 Probe Master (Roche, Shanghai). PCR products were confirmed by agarose gel electrophoresis and melting temperature.

\section{Western-blot}


Proteins were extracted from $661 \mathrm{~W}$ cell lysates using the RIPA buffer containing cocktails of protease inhibitors and phosphatase inhibitors and the protein concentration was determined by BCA assay (Solarbio, Beijing, Cat\# PC0020). Equal quantities of protein (40 $\mu \mathrm{g}$ ) prepared in RIPA buffer were applied to 10-12\% SDS-PAGE and transferred onto Nitrocellulose membranes (Pall, New York, Cat\#66485). The separation of different proteins was confirmed by Ponceau staining. The membranes were blocked with $5 \%$ non-fat milk for $1 \mathrm{~h}$ at room temperature and incubated with primary antibody anti-CD59 (1:1000, Abcam, Shanghai, Cat\#ab9183, RRID:AB_307054), anti-CD47 (1:1000, Abcam, Cat\# ab175388, RRID: AB_2813735) and anti- $\beta$-actin (1:5000, Cell Signaling Technology, Shanghai, Cat\# 4970, RRID:AB_2223172) at $4{ }^{\circ} \mathrm{C}$ overnight respectively, followed by incubation with secondary antibodies conjugated with IRDye (IRDye800CW-goat anti-rabbit IgG: 1:10000, Cat\# 926-32232, RRID:AB_10806644, IRDye680CW-goat anti-Mouse IgG :1:10000, Cat\# 926-68070, RRID:AB_10956588, all from LI-COR, Nebraska, NE) respectively at room temperature for $2 \mathrm{~h}$. Blotting images were captured using the odyssey infrared imaging system(Li-COR, Nebraska). The density analysis was performed by Image J (National Institutes of Health, USA).

\section{Immunofluorescence}

Mouse eyes were fixed in 2\% PFA for $2 \mathrm{~h}$ and embedded in OCT and cryosectioned with $10 \mu \mathrm{m}$ thickness and processed for immunofluorescent staining. Cryosections from retinal explants were fixed with $2 \%$ PFA from 10 min and processed for immunofluorescent staining. In addition, retinal sections from our previous studies including 7 days retinal detachment (subretinal injection of hyaluronic acid, Chen et al ARVO abstract, 2018) and 25 days experimental autoimmune uveoretinitis (Chen et al. 2018) were also used to examine the expression of CD59a and CD47 in diseased retina. 
Mouse eye sections were blocked with $10 \%$ goat serum $+2 \%$ BSA with $0.1 \%$ Triton-X 100 in PBS for $1 \mathrm{~h}$ at room temperature, then incubated with primary antibodies mouse anti-CD59 (MEM-43, 1:100,Cat\# ab9183, RRID: AB307054) and rabbit anti-arrestin C (1:100, ab180912, RRID:AB2813738) or rabbit anti-mouse CD47 (1:100, Cat\#ab175388, RRID: AB2813735) and mouse anti-Rhodopsin (Rho4D2, 1:100, ab98887, RRID: AB10696805) (all from Abcam) respectively at $4{ }^{\circ} \mathrm{C}$ overnight. After thorough washes, the samples were incubated with secondary antibodies, including Alexa Fluor 647-conjugated goat anti-mouse IgG (1:500, Cat\#A-21235, RRID: AB141693) and Alexa Fluor 488-conjugated goat anti-rabbit IgG (1:500, Cat\#A-11008, RRID: AB143165) or Alexa Fluor 488-conjugated goat anti-mouse IgG (1:500, Cat\#A-11001, RRID:AB2534069) and Alexa Fluor 594 conjugated goat anti-rabbit IgG (1:500, Cat\#A-11012, RRID:AB141359) (all from Invitrogen, Carlsbad, CA) for $1 \mathrm{~h}$ at room temperature. All samples were imaged using the Zeiss LSM 880 confocal microscope (Zeiss, Shanghai).

\section{Immunohistochemistry}

The fixed human eye balls were embedded in paraffin as previously described (Li et al. 2016). The paraffin blocks were cut using microtome with $5 \mu \mathrm{m}$ thickness and the sections were mounted on glass slides. After dewaxing and rehydration (Stumptner et al. 2016), samples were further processed for immunohistochemistry.

Samples were immersed in sodium citrate buffer (10mM sodium citrate, $0.05 \%$ Tween 20, pH 6.0) and incubated for 6 min in pressure cooker. After unartificial cooling, the sections were blocked with alkaline phosphatase inhibitor (DingGuo, Beijing, Cat\#DGSP-A-KIT-1) for 30 min followed by $10 \%$ goat serum and $2 \%$ BSA for $1 \mathrm{~h}$ at room temperature. Samples were then incubated overnight with primary antibodies (mouse anti-CD59, 1:500, Abcam, Cat\#ab9183; and rabbit antiCD47, 1:500, Abcam, Cat\#ab175388). After thorough washes, samples were incubated with the appropriate biotinylated secondary antibodies (goat anti-mouse IgG H\&L or goat anti-rabbit IgG 
H\&L: Cat\#DGSP-A-KIT-3 ) and the protein solution of anti-biotin conjugated with alkaline phosphatase from the Biotin SP-AP immunohistochemical kit (Cat\# DGSP-A-KIT-4) for 30 min. Color was developed using the BCIP/NBT alkaline phosphatase (AP) substrate (Cat\#AR-0632 ) and counter-stained with Nuclear Fast Red (Cat\#AR-0771) (all from DingGuo).

\section{Statistical analysis}

All experiments were repeated at least three times. Data were analysed using the GraphPad Prism software (version 5, San Diego, CA). Data were presented as mean \pm SD. Unpaired Student's ttest was used to compare the difference between the treatment group and non-treated control group. The density of Western-blot was normalized to the non-treatment control group. A p value $<0.05$ was regarded as statistically significant.

\section{Results}

\section{Immune regulatory gene expression in retinal cells in single cell sequencing}

Using known gene markers (Table 1, Fig. 1A) major retinal cells were identified in the ScRNAseq datasets published by Heng et al (Heng et al. 2019) (GSM3580725 and GSM3580727). The cells include rods (6730 cells, 61.32\%), cones (682 cells, 5.72\%), bipolar cells (1949 cells, 17.76\%), amacrine cells (1059 cells, 9.65\%), Müller glia (344 cells, 3.13\%), retinal ganglion cells (48 cells, $0.44 \%$ ), horizontal cells (33 cells, $0.30 \%$ ), microglia ( 24 cells, $0.22 \%$ ), endothelial cells (78 cells, $0.71 \%)$ and pericytes (29 cells, $0.26 \%)$ (Fig. 1B).

The immune regulatory genes including CD200, CD47, SIRP $\alpha$, CX3CL1, CX3CR1, TGF $\beta$, Thbs1 and various complement related genes were detected in retinal cells. CD200 was highly expressed by endothelial cells followed by ganglion cells, horizontal cells, pericytes, and amacrine cells (Fig. 
2A). CX3CL1 was highly expressed by ganglion cells, amacrine cells and horizontal cells; whereas its receptor CX3CR1 was expressed almost exclusively in microglia (Fig. 2A). CD47 was expressed at by almost all retinal cells and its receptor SIPR $\alpha$ was mainly expressed by microglia (Fig. 2A). Further analysis using the violin plot showed that all horizontal cells and the majority of ganglion cells express CD47. A small population of rods express also high levels of CD47 (Fig. 2B). Three TGF $\beta$ genes were detected in the retina with the highest being TGF $\beta 2$ by Müller cells and bipolar cells, followed by TGF $\beta 1$, which was detected predominately in microglia (Fig. 2A). IL-34 was detected in pericytes and ganglion cells (Fig. 2A).

Twelve complement related genes were detected in retinal cells (Fig. 2A). In general, the expression levels of genes involved in complement inhibition (complement factor $\mathrm{H}(\mathrm{Cfh}), \mathrm{Cr} 11$ (Crry), CD59a, Serping1) were higher than those involved in complement activation (C1ra, C2, C4b, Masp2) (Fig. 2A). CFH was highly expressed by pericytes, microglia and endothelial cells. Pericytes also expressed high levels of Serping1 (Fig. 2A). Interestingly, CD59a was highly expressed by retinal neurons, including cones, horizontal cells, and ganglion cells (Fig. 2A). Further analysis using the violin plot showed that a small population of rods, bipolar cells and amacrine cells also express CD59a (Fig. 2C). Complement receptor C5ar1 was predominately expressed by microglia (Fig. 2A).

\section{Immune regulatory gene expression in photoreceptors}

Under normal physiological conditions, the outer retina (from outer nuclear layer to subretinal space) is devoid of immune cells and considered as an immune privileged site (Forrester et al. 2008, Forrester \& Xu 2012). To understand if photoreceptors contribute to this immune privilege, the profile of immune regulatory gene expression in cones and rods was examined in more detail. Fig. 3 shows that $34.16 \%$ of cones (Fig. 3A) and $2.76 \%$ rods (Fig. 3B) express high levels of CD59a. Low levels of CD59a were also detected in the remaining cone photoreceptors (Fig. 2A). High levels of CD47 were detected in $17.01 \%$ of cones (Fig. 3A) and 3.63\% of rods (Fig. 3B). 
Other genes, including CD200, Sirpa, CX3CL1, Tgfb2 and Cr11 (Crry) were also detected in small populations of rods and cones (Fig. 3).

To confirm the scRNA-seq results, we conducted real-time RT-PCR in $661 \mathrm{~W}$ cells, a mouse cone cell line (Tan et al. 2004), photoreceptors dissected from human eye sections and human retinoblastoma cell line WERI-Rb1, which is known to be able to differentiate into retinal neurons including photoreceptors (Liu et al. 2017). CD200, SIPR $\alpha$, CD47, CX3CL1, TGF $\beta$, and CD59a were all detected in $661 \mathrm{~W}$ cells (Fig. 4A). Other complement related genes including Cfh, C1r and $\mathrm{C} 1 \mathrm{~s}$ were also detected in $661 \mathrm{~W}$ cells although at low levels (Fig. 4A). The expression of the immune regulatory genes including Sirpa, CD47, Thbs1 and CD59a was further confirmed in WERI-Rb1 cells (Fig. 4B) and human photoreceptors (Fig. 4C).

\section{CD59 and CD47 protein expression in the retina}

Among all tested genes in our study, CD59a and CD47 were the most highly expressed genes in photoreceptors (Fig. 3). We, therefore, further investigated these two genes in the rest of the study. Arrestin C was widely expressed by retina cells, including GCL, INL, IPL, ONL and IS (Fig. 5A). Extensive expression of CD59a in ONL and IS/OS of photoreceptors was detected in normal retina (Fig. 5A). Outer limiting membrane (arrows, Fig. 5A) and photoreceptor out segments, in particular, express high levels of CD59a (Fig. 5A). Discrete immunoreactivity of CD59a was also detected in ganglion cell layer, IPL and OPL (arrowheads, Fig. 5A; arrows, Fig.s 5B, 5C). The immunoreactivity of CD59a in GCL, IPL, OPL and photoreceptors was also noted in human retina (Fig. 5E). Like the mouse retina, the outer limiting membrane and inner segments of photoreceptors were stained positive for CD59a (Fig. 5E). Vascular endothelial cells also appear to be positive for CD59a (arrow in Fig. 5E).

CD47 was detected in all retinal layers of the mouse eyes (Fig. 5F). Ganglion cells, in particular, express high levels of CD47 (Fig. 5F). Further examination in high magnification images showed 
that many photoreceptors also express CD47 (arrows in Fig. 5G). Immunohistochemistry of human eyes showed CD47 immunoreactivity in GCL, IPL, OPL and the inner segment of photoreceptors (Fig. 5I). Discrete CD47 expression was also detected in ONL (Fig. 5I).

\section{Expression of CD59 and CD47 in photoreceptors under pathological conditions}

To understand how the expression of CD59a and CD47 in photoreceptors may be affected in disease conditions, $661 \mathrm{~W}$ cells were treated with TNF $\alpha(20 \mathrm{ng} / \mathrm{ml})$, LPS (100 ng/ml), IL-4 (20 $\mathrm{ng} / \mathrm{ml})$, hypoxia $\left(5 \% \mathrm{O}_{2}\right)$, or $\mathrm{H}_{2} \mathrm{O}_{2}(200 \mu \mathrm{M})$ for $18 \mathrm{~h}$. The expression of CD59a and CD47 mRNA was examined by real-time quantitative RT-PCR. Our results show that the expression of CD59a mRNA was upregulated by TNF $\alpha(t=3.75, \mathrm{p}=0.02)$, hypoxia $(\mathrm{t}=7.29, \mathrm{p}=0.002)($ Fig. $6 \mathrm{~A})$ and $\mathrm{H}_{2} \mathrm{O}_{2}$ $(\mathrm{t}=8.38, \mathrm{p}=0.001$, Supplementary data 2$)$. LPS and IL-4 did not significantly affect CD59a expression in 661W cells (Fig. 6A). Western blot detected increased levels of CD59a following LPS $(\mathrm{t}=13.25 ; \mathrm{p}=0.0002)$ and IL-4 $(\mathrm{t}=5.97 ; \mathrm{p}=0.004)$ treatment (Fig. 6B). However, the increment in CD59a protein following $\mathrm{TNF} \alpha$ or hypoxia treatments did not reach statistical significance, probably due to large variation (Fig. 6B).

The expression of CD47 mRNA was upregulated by LPS ( $\mathrm{t}=3.71, \mathrm{p}=0.021)$, IL-4 $(\mathrm{t}=3.35$, $\mathrm{p}=0.029)$, hypoxia $(\mathrm{t}=21.89 ; \mathrm{p}<0.0001)$, and $\mathrm{H}_{2} \mathrm{O}_{2}(\mathrm{t}=9.18, \mathrm{p}=0.0008$, Supplementary data 2$)$, but not TNF $\alpha(t=2.28 ; p=0.085)$ (Fig. 6C). Western blot showed mild but statistically insignificant upregulation of CD47 in LPS and IL-4 treated cells (Fig. 6D). The mismatch between mRNA and protein expression following cytokine/hypoxia treatment suggests additional control of CD47 expression at the mRNA-to-protein translational level in $661 \mathrm{~W}$ cells and this warrants further investigation.

Immunostaining of CD59a and arrestin $\mathrm{C}$ in the detached retina showed massive reduction in arrestin C expression but increased expression of CD59a in photoreceptors, particularly in the OPL and IS/OS of photoreceptors compared to that in healthy retina (Fig. 7A). Upregulation of 
CD59a in photoreceptors and cells of the INL was observed in retinal explants cultured for $6 \mathrm{~h}$ (Fig. 7A). In the EAU retina, CD59a was expressed predominately by active immune cells, including infiltrating cells (arrows, Fig. 7A).

The expression of CD47 was reduced in GCL but increased in OPL and photoreceptors in the detached retina and retinal explants cultured for 6h (Fig. 7B). CD47 was also observed in Müller cells in the detached retina and cultured retinal explants (Fig. 7B). In the EAU retina, the expression of CD47 was reduced in photoreceptors, but increased in OPL (Fig. 7B). Vitreous infiltrating cells also express high levels of CD47 (Fig. 7B).

\section{Regulation of BV2 microglial activation by $661 \mathrm{~W}$}

To test the immune regulatory function of photoreceptors, BV2 microglia were co-cultured with $661 \mathrm{~W}$ cells. The microglial cells were then isolated and their pro-/anti-inflammatory gene expression was examined. $661 \mathrm{~W}$ cells increased $\mathrm{IL}-1 \beta$ expression in naïve $(t=8.95, \mathrm{p}=0.0009)$ and IL-14 ( $\mathrm{t}=5.92, \mathrm{p}=0.004)$ treated BV2 cells (Fig. 8A), but significantly reduced ILP+IFN $\gamma$-induced IL-6 expression ( $\mathrm{t}=8.54, \mathrm{p}=0.001$, Fig. 8B). Importantly, the expression of arginase- 1 in naïve $(\mathrm{t}=12.03, \mathrm{p}=0.0003)$ or LPS $+\operatorname{IFN} \gamma(\mathrm{t}=6.24, \mathrm{p}=0.003)$ or IL-4 $(\mathrm{t}=3.7, \mathrm{p}=0.02)$ treated cells were all significantly increased by $661 \mathrm{~W}$ cells (Fig. 8C).

\section{Discussion}

In this study, using publically available retinal RNA single cell RNA sequence datasets, we show that retinal neurons, including ganglion cells, horizontal cells, amacrine cells and photoreceptors express various immune regulators. Specifically, CD200, CX3CL1 and CD47 are expressed predominately by ganglion cells, amacrine cells and horizontal cells; whereas, their receptors CX3CR1 and SIRPa are expressed by microglia. We also found that CD47 and CD59a are predominately expressed by cone photoreceptor, horizontal cells and ganglion cells. Our results highlight the importance of retinal neurons in regulating local immune activation. 
Retinal microglia are distributed in the ganglion cell layer, IPL and OPL under normal healthy conditions (Karlstetter et al. 2015, Chen \& Xu 2015) (Fig. 8). A recent study suggests that microglia in different retinal layers may function differently and majority of the microglia in the inner retina are IL-34-dependent and may critically control neuronal function (O'Koren et al. 2019). Neurons express molecules such as CX3CL1, CD47 and CD200 that negatively regulate microglial activation (Langmann 2007) and loss of these pathways can lead to uncontrolled inflammation and retinal degeneration (Chen et al. 2011, Dick et al. 2003). Our results suggest that microglia in the IPL may be controlled predominately by ganglion and added by amacrine cells; whereas the activation of microglia in the OPL may be regulated mainly by horizontal cells and photoreceptors (Fig. 9). Bipolar cells express low levels of TGF $\beta 2$ and CD47 and may also participate in the regulatory process (Fig. 8). IL-34 is detected in ganglion cells and pericytes in the healthy retina (Fig. 2A). This pattern of expression is in line with their role in maintaining IPL microglia function reported by O'Koren et al (O'Koren et al. 2019). IL-34 is known to promote microglial proliferation and neuroprotection in the CNS (Mizuno et al. 2011). The role of IL-34 in retinal microglial activation, proliferation and function warrant further investigation. It must be noted that the number of retinal ganglion cells (RGC) and horizontal cells (HC) identified from the sc-RNAseq study was very low and the results may not fully represent the true expression profile of the immune regulators in these cells, however, the expression profile of the immune regulators in photoreceptors (rods and cones), bipolar cells and amacrine cells are supported by sufficient cell numbers.

In this study, CD59a and CD47 were also detected in a large number of photoreceptors and 661W suppressed pro-inflammatory (IL-6) and enhanced anti-inflammatory (Arg-1) gene expression in BV2 cells suggesting that photoreceptors may also play an important role in regulating immune activation in the OPL and subretinal space. CD47 is a transmembrane protein that partners with other membrane integrins and can bind ligands SIPR $\alpha$ and thrombospondin-1 (TPS1)(Veillette \& 
Chen 2018). SIRPa is an inhibitory receptor expressed predominately on microglia in the retina (Fig. 2A). Binding of CD47 to SIRPa recruits inhibitory molecules SHP-1/2 that prevent cell activation (Barclay \& Van den Berg 2014). In addition, CD47 can act as a “don’t eat me” signal to macrophages and blocking the CD47-SIRPa pathway has shown promise as a new way to treat cancer (Veillette \& Chen 2018). Retinal neurons may use similar strategies to avoid being phagocytosed by active microglia and infiltrating phagocytes under disease conditions. CD47 can also influence inflammation and angiogenesis through interacting with TSP-1. A recent study has shown that TSP-1-mediated CD47 activation is critically involved in the elimination of infiltrating subretinal microglia and macrophages (Calippe et al. 2017).

The expression of CD47 in photoreceptors is affected by inflammatory cytokines and hypoxic conditions. CD47 expression in photoreceptors was increased in the detached retina but reduced in EAU retina. The former represents hypoxic conditions; whereas the latter is an acute inflammation. Microglia and macrophages can infiltrate the outer retina under disease conditions. Down-regulation of CD47 in photoreceptors in EAU may be a result of acute inflammation at this site and may sensitise photoreceptors to inflammatory insults. The upregulation of CD47 by photoreceptors in retinal detachment may be a mechanism to control inflammation in the outer retina.

Activation of the complement system may damage the retina by (a) promoting inflammation (e.g., via $\mathrm{C} 3 \mathrm{a}$ or $\mathrm{C} 5 \mathrm{a}$ ) and (b) directly killing host cells (via the membrane attack complex, MAC, or C5b-9) (Zipfel et al. 2007). How complement activation in the neuroretina is regulated is not fully understood. Cfh, the key inhibitor of the alternative pathway of the complement system, is expressed by microglia, pericytes and endothelial cells (Fig. 2A), but not photoreceptors, suggesting that it may safeguard the inner retina from alternative pathway-mediated complement attack. CD59, on the other hand, is expressed at high levels in cones and rods (Figs. 2\&3). CD59 is a glycosylphosphatidylinositol (GPA) anchored membrane protein that prevents the binding of 
C9 to C5b-8 complex, thereby protects host cells from complement attack (Farkas et al. 2002). In mouse, CD59a is the primary regulator of MAC assembly (Baalasubramanian et al. 2004). CD59 has been detected in mouse (Bora et al. 1993) and human (Vogt et al. 2006) retina, and its retinal expression increases with age (Faber et al. 2012). In the healthy retina $34 \%$ of cones and $2.7 \%$ of rods express high levels of C59a, suggesting that photoreceptors may critically control complement activation in the out layers of the retina.

CD59 also has important complement-independent roles, including the regulation of T/B and NK cell activation, cell migration and proliferation (Kimberley et al. 2007). Furthermore, CD59 can detach from the cell membrane and the soluble form of CD59 (sCD59) is released into body fluids (e.g., urine, milk, serum, plasma and cerebrospinal fluid)(Vakeva et al. 2000, Ghosh et al. 2013, Zelek et al. 2019, Budding et al. 2016). It has been reported that the levels of sCD59 in the cerebrospinal fluid nearly doubled those in the plasma in people with demyelinating disorders (Zelek et al. 2019), suggesting an intrathecal origin of sCD59. Although the biological function of sCD59 is largely unknown, previous studies have shown that AAV-mediated delivery of sCD59 can attenuate retinal pathologies in diabetic mice (Adhi et al. 2013), laser-induced choroidal neovascularization (Cashman et al. 2011), and experimental autoimmune uveoretinitis (Kumar et al. 2018). The CD59-expressing neurons may participate in retinal immune regulation beyond complement activation.

A major limitation of our study is the lack of functional testing on these retinal neuron-specific inhibitory molecules. Using neuron (e.g., photoreceptor) specific CD59a or CD47 gene knockout mice help to understand the role of these molecules in retinal neurons and in immune regulation.

\section{Conclusion}

Retinal neurons, including ganglion cells, amacrine cells, horizontal cells and photoreceptors express various immune regulators that may critically control retinal microglia and complement 
activation. Based on our results, we believe that photoreceptors may also participate in retinal immune regulation, for example by expressing CD47 and CD59a. Together with retinal pigment epithelial cells, photoreceptors may critically control the immune suppressive microenvironment of outer retinal layers and subretinal space. Further understanding the immune regulatory role of retinal neurons including photoreceptors will be important to develop better treatment strategies for inflammatory and degenerative retinal diseases.

\section{Ethics Statement}

This study was conducted in accordance with the recommendations of the ARVO Statement for the Use of Animals in Ophthalmic and Vision Research. The protocol was approved by the Animal Welfare and Ethical Review Body at Queen's University Belfast (PPL 2773).

\section{Author contributions}

JL, MT, KH, CL and XD conducted experiments and acquired data. MC and HX developed the conception and designed the study. JL, MT, MC and HX interpreted data and wrote the manuscript. KH reviewed the manuscript.

\section{Conflict of Interest Statement}

The authors declare that the research was conducted in the absence of any commercial or financial relationships that could be construed as a potential conflict of interest.

\section{Funding support}

This project has received funding from (1) Aier Eye Hospital Group, (2) the European Union's Horizon 2020 research and innovation programme under the Marie Skłodowska-Curie grant agreement No 722717, (3) National Natural Science Foundation of China (81700827) and (4) Natural Science Foundation of Hunan Province, China (2018JJ3002). 


\section{Acknowledgement}

The authors thank Dr Congjian Zhao and Mrs Haiping Que for technical advice.

\section{Data Accessibility}

The retinal single-cell RNA sequencing (scRNA-seq) datasets used in this study were downloaded from the GEO database from https://www.ncbi.nlm.nih.gov/. Sample ID: GSM3580725 and GSM3580727. 


\section{Reference}

Adhi, M., Cashman, S. M. and Kumar-Singh, R. (2013) Adeno-associated virus mediated delivery of a non-membrane targeted human soluble CD59 attenuates some aspects of diabetic retinopathy in mice. PloS One, 8, e79661.

Arcuri, C., Mecca, C., Bianchi, R., Giambanco, I. and Donato, R. (2017) The Pathophysiological Role of Microglia in Dynamic Surveillance, Phagocytosis and Structural Remodeling of the Developing CNS. Front Mol Neurosci, 10, 191.

Augustine, J., Pavlou, S., Ali, I., Harkin K., Ozaki, E., Campbell, M., Stitt, A.W., Xu, H., Chen, M. (2019) IL-33 deficiency causes persistent inflammation and severe neurodegeneration in retinal detachment. J Neuroinflammation 16 (1), 251

Baalasubramanian, S., Harris, C. L., Donev, R. M., Mizuno, M., Omidvar, N., Song, W. C. and Morgan, B. P. (2004) CD59a is the primary regulator of membrane attack complex assembly in the mouse. $J$ Immunol, 173, 3684-3692.

Barclay, A. N. and Van den Berg, T. K. (2014) The interaction between signal regulatory protein alpha (SIRPalpha) and CD47: structure, function, and therapeutic target. Annu Rev Immunol, 32, 25-50.

Bora, N. S., Gobleman, C. L., Atkinson, J. P., Pepose, J. S. and Kaplan, H. J. (1993) Differential expression of the complement regulatory proteins in the human eye. Invest Ophthal \& Vis Sci, 34, 3579-3584.

Budding, K., van de Graaf, E. A., Kardol-Hoefnagel, T. et al. (2016) Soluble CD59 is a Novel Biomarker for the Prediction of Obstructive Chronic Lung Allograft Dysfunction After Lung Transplantation. Sci Rep, 6, 26274.

Butler, A., Hoffman, P., Smibert, P., Papalexi, E. and Satija, R. (2018) Integrating single-cell transcriptomic data across different conditions, technologies, and species. Nat Biotechnol, 36, 411-420.

Calippe, B., Augustin, S., Beguier, F. et al. (2017) Complement Factor H Inhibits CD47-Mediated Resolution of Inflammation. Immunity, 46, 261-272. 
Cashman, S. M., Ramo, K. and Kumar-Singh, R. (2011) A non membrane-targeted human soluble CD59 attenuates choroidal neovascularization in a model of age related macular degeneration. PloS One, $\mathbf{6}$, e19078.

Chan, C. C., Tuo, J., Bojanowski, C. M., Csaky, K. G. and Green, W. R. (2005) Detection of CX3CR1 single nucleotide polymorphism and expression on archived eyes with age-related macular degeneration. Histol Histopathol, 20, 857-863.

Chen, M., Forrester, J. V. and Xu, H. (2011) Dysregulation in retinal para-inflammation and age-related retinal degeneration in CCL2 or CCR2 deficient mice. PloS One, 6, e22818.

Chen, M., Luo, C., Zhao, J., Devarajan, G. and Xu, H. ( (2019) ) Immune regulation in the aging retina. Prog Retin Eye Res, 69, 159-172.

Chen, M. and Xu, H. (2015) Parainflammation, chronic inflammation, and age-related macular degeneration. J Leukoc Biol, 98, 713-725.

Chen, M., Zhao, J., Ali, I. H. A., Marry, S., Augustine, J., Bhuckory, M., Lynch, A., Kissenpfennig, A. and Xu, H. (2018) Cytokine Signaling Protein 3 Deficiency in Myeloid Cells Promotes Retinal Degeneration and Angiogenesis through Arginase-1 Up-Regulation in Experimental Autoimmune Uveoretinitis. Am J Path, 188, 1007-1020.

Chu, Y., Jin, X., Parada, I., Pesic, A., Stevens, B., Barres, B. and Prince, D. A. (2010) Enhanced synaptic connectivity and epilepsy in C1q knockout mice. PNAS USA, 107, 7975-7980.

Dick, A. D., Carter, D., Robertson, M., Broderick, C., Hughes, E., Forrester, J. V. and Liversidge, J. (2003) Control of myeloid activity during retinal inflammation. J Leukoc Biol, 74, 161-166.

Eandi, C. M., Messance, H. C., Augustin, S., Dominguez, E., Lavalette, S., Forster, V., ... Sennlaub, F. (2016). Subretinal mononuclear phagocytes induce cone segment loss via IL-1 $\beta$. ELife, 5(JULY), 116. https://doi.org/10.7554/eLife.16490 
Faber, C., Williams, J., Juel, H. B., Greenwood, J., Nissen, M. H. and Moss, S. E. (2012) Complement factor $\mathrm{H}$ deficiency results in decreased neuroretinal expression of $\mathrm{Cd} 59 \mathrm{a}$ in aged mice. Invest Ophthalmol \& Vis Sci, 53, 6324-6330.

Farkas, I., Baranyi, L., Ishikawa, Y., Okada, N., Bohata, C., Budai, D., Fukuda, A., Imai, M. and Okada, H. (2002) CD59 blocks not only the insertion of C9 into MAC but inhibits ion channel formation by homologous C5b-8 as well as C5b-9. J Physiol, 539, 537-545.

Forrester, J. V. and Xu, H. (2012) Good news-bad news: the Yin and Yang of immune privilege in the eye. Front Immunol, 3, 338.

Forrester, J. V., Xu, H., Lambe, T. and Cornall, R. (2008) Immune privilege or privileged immunity? Mucosal Immunol, 1, 372-381.

Ghosh, P., Sahoo, R., Vaidya, A. et al. (2013) A specific and sensitive assay for blood levels of glycated CD59: a novel biomarker for diabetes. Am J Hematol, 88, 670-676.

Hafemeister, C. \& Satija, R. (2019) Normalization and variance stabilization of single-cell RNA-seq data using regularized negative binomial regression. bioRxiv 576827. doi:10.1101/576827

Heng, J. S., Rattner, A., Stein-O'Brien, G. L., Winer, B. L., Jones, B. W., Vernon, H. J., Goff, L. A. and Nathans, J. (2019) Hypoxia tolerance in the Norrin-deficient retina and the chronically hypoxic brain studied at single-cell resolution. PNAS USA, 116, 9103-9114.

Howell, G. R., Macalinao, D. G., Sousa, G. L. et al. (2011) Molecular clustering identifies complement and endothelin induction as early events in a mouse model of glaucoma. J Clin Invest, 121, 1429-1444.

Karlstetter, M., Scholz, R., Rutar, M., Wong, W. T., Provis, J. M. and Langmann, T. (2015) Retinal microglia: just bystander or target for therapy? Prog Retin Eye Res, 45, 30-57.

Kimberley, F. C., Sivasankar, B. and Paul Morgan, B. (2007) Alternative roles for CD59. Mol Immunol, 44, 73-81. 
Kumar, B., Cashman, S. M. and Kumar-Singh, R. (2018) Complement-Mediated Activation of the NLRP3 Inflammasome and Its Inhibition by AAV-Mediated Delivery of CD59 in a Model of Uveitis. Mol Ther, 26, 1568-1580.

Langmann, T. (2007) Microglia activation in retinal degeneration. J Leukoc Biol. 81, 1345-1351.

Li, H., Zhu, C., Wang, B. et al. (2016) 17beta-Estradiol Protects the Retinal Nerve Cells Suppressing TLR2 Mediated Immune-Inflammation and Apoptosis from Oxidative Stress Insult Independent of PI3K. $J$ Mol Neurosci, 60, 195-204.

Liu, Y., Hu, H., Liang, M. et al. (2017) Regulated differentiation of WERI-Rb-1 cells into retinal neuronlike cells. Int J Mol Med, 40, 1172-1184.

Mizuno, T., Doi, Y., Mizoguchi, H., Jin, S., Noda, M., Sonobe, Y., Takeuchi, H. and Suzumura, A. (2011) Interleukin-34 selectively enhances the neuroprotective effects of microglia to attenuate oligomeric amyloid-beta neurotoxicity. Am J Pathol, 179, 2016-2027.

O'Koren, E. G., Yu, C., Klingeborn, M. et al. (2019) Microglial Function Is Distinct in Different Anatomical Locations during Retinal Homeostasis and Degeneration. Immunity, 50, 723-737.e727.

Schafer, D. P., Lehrman, E. K. and Stevens, B. (2013) The "quad-partite" synapse: microglia-synapse interactions in the developing and mature CNS. Glia, 61, 24-36.

Stumptner, C., Gogg-Kamerer, M., Viertler, C., Denk, H. and Zatloukal, K. (2016) Immunofluorescence and Immunohistochemical Detection of Keratins. Methods Enzymol, 568, 139-162.

Szepesi, Z., Manouchehrian, O., Bachiller, S. and Deierborg, T. (2018) Bidirectional Microglia-Neuron Communication in Health and Disease. Frontiers in cellular neuroscience, 12, 323.

Tan, E., Ding, X. Q., Saadi, A., Agarwal, N., Naash, M. I. and Al-Ubaidi, M. R. (2004) Expression of conephotoreceptor-specific antigens in a cell line derived from retinal tumors in transgenic mice. Invest Ophthalmol Vis Sci, 45, 764-768.

Taylor, A. W. and Ng, T. F. (2018) Negative regulators that mediate ocular immune privilege. J Leukoc Biol. 18;103: 1179-1187. 
Vakeva, A., Lehto, T., Takala, A. and Meri, S. (2000) Detection of a soluble form of the complement membrane attack complex inhibitor CD59 in plasma after acute myocardial infarction. Scand $J$ Immunol, 52, 411-414.

Veillette, A. and Chen, J. (2018) SIRPalpha-CD47 Immune Checkpoint Blockade in Anticancer Therapy. Trends Immunol, 39, 173-184.

Vogt, S. D., Barnum, S. R., Curcio, C. A. and Read, R. W. (2006) Distribution of complement anaphylatoxin receptors and membrane-bound regulators in normal human retina. Exp Eye Res, 83, 834-840.

$\mathrm{Xu}, \mathrm{H}$. and Chen, M. (2016) Targeting the complement system for the management of retinal inflammatory and degenerative diseases. Eur J Pharmacol, 787, 94-104.

Xu, H., Chen, M. and Forrester, J. V. (2009) Para-inflammation in the aging retina. Prog Retin Eye Res, 28, 348-368.

Xu, H., Manivannan, A., Crane, I., Dawson, R., Liversidge, J. (2008) Critical but divergent roles for CD62L and CD44 in directing blood monocyte trafficking in vivo during inflammation. Blood 112 (4), 11661174

Zelek, W. M., Watkins, L. M., Howell, O. W. et al. (2019) Measurement of soluble CD59 in CSF in demyelinating disease: Evidence for an intrathecal source of soluble CD59. Mult Scler, 25, 523-531.

Zipfel, P. F., Mihlan, M. and Skerka, C. (2007) The alternative pathway of complement: a pattern recognition system. Adv Exp Med Biol, 598, 80-92. 


\section{Figures and Legends}
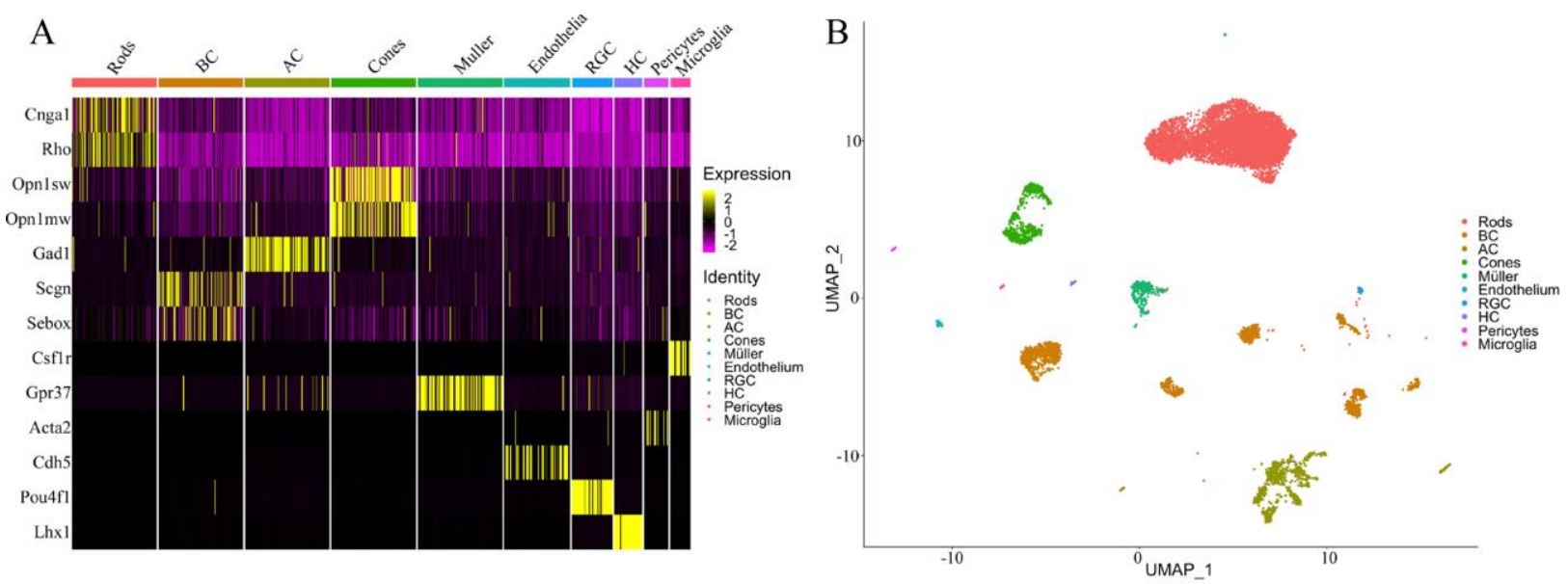

Figure 1. Identification of different types of retinal cells using scRNA-seq technique. Two scRNA-seq datasets from online resource (Heng et al. PNAS. 2019,116(18):9103-9114) were merged in to one genes $\times$ cells counts matrix. The Seurat $R$ package (version 3.0.0) was used to analyse the merged single-cell dataset. (A) Heat map showing gene expression profile of different types of retinal cells. (B) Cluster analysis of retinal cells visualized in a UMAP representation. UMAP - uniform manifold approximation and projection.
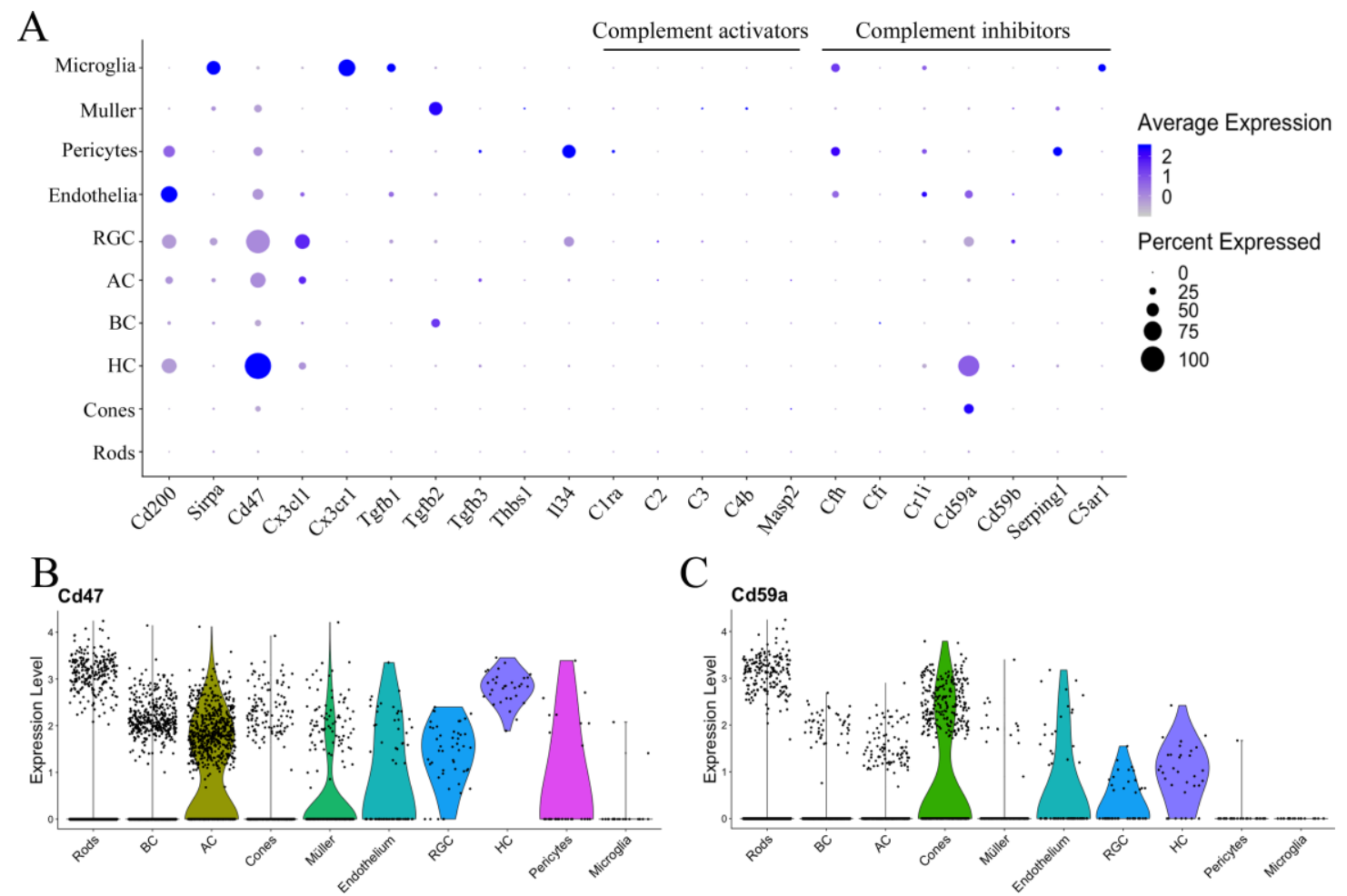

Figure 2. Immune regulator gene expression in different types of retinal cells. scRNA-seq data were used to analyse the expression levels of different immune regulators in retinal cells. (A) The expression profile (percentage of cell and average expression levels) of various immune regulatory genes expression in different retinal cells. (B) A violin plot showing the expression 
levels of CD47 and the number of CD47 $7^{+}$cells in different types of retinal cells. (C) A violin plot showing the expression levels of CD59a and the number of CD59a ${ }^{+}$cells in different types of retinal cells.
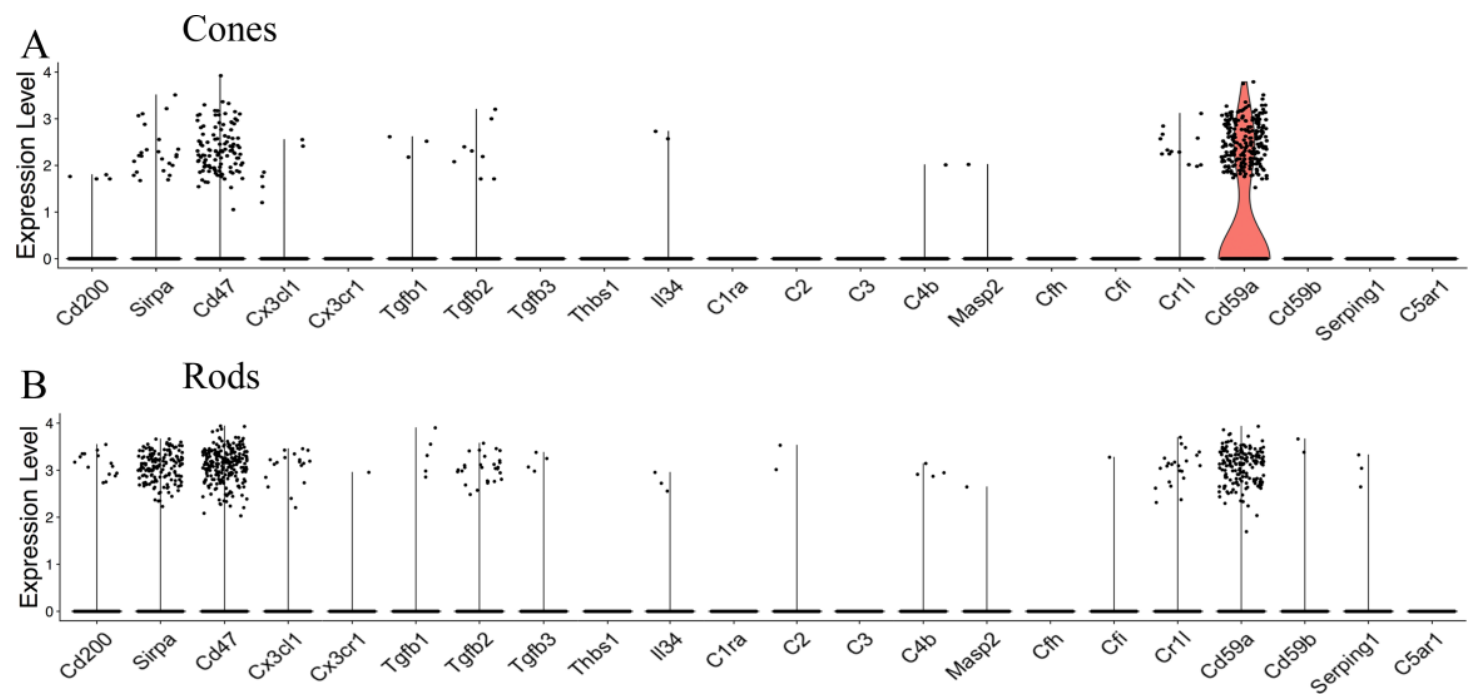

Figure 3. Immune regulatory gene expression in cones and rods. scRNA-seq data were used to analyse expression levels of different immune regulators in cone and rod photoreceptors. (A \& $\mathrm{B})$ The expression profile (percentage of cell and average expression levels) of various immune regulatory genes expression in cone (A) and rod (B) photoreceptors.
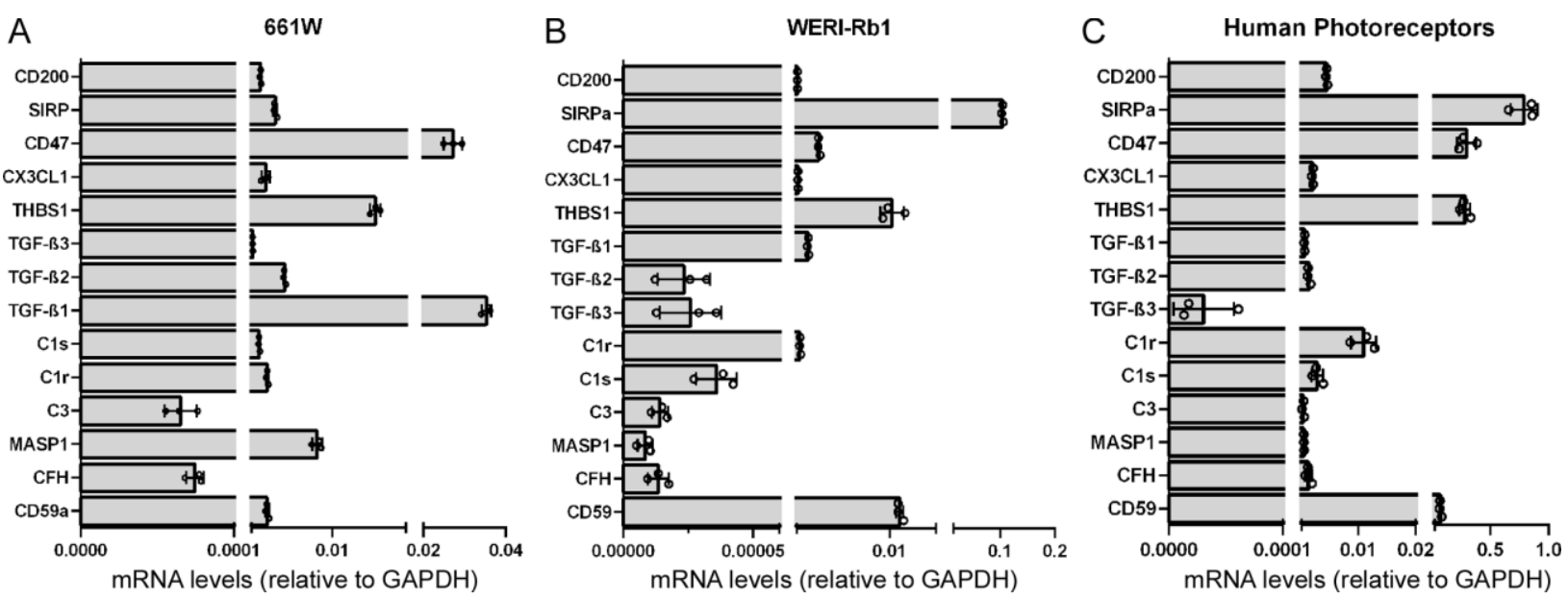

Figure 4. Real-time RT-PCR analysis of immune regulatory gene expression in photoreceptors. The expression of immune regulatory genes including CD200, SIRPa, CD47, CX3CL1, Thbs, TGF $\beta 1-3$, CFH, CD59 and complement genes C1s, C1r, and C3 in 661W (A), WERI-Rb1 (B) and microdissected photoreceptors (C) were examined by real-time quantitative RT-PCR. Data presented are relative mRNA levels compared with GAPDH. Mean $\pm \mathrm{SD}, \mathrm{N}=3$. Experiments were repeated three times. 

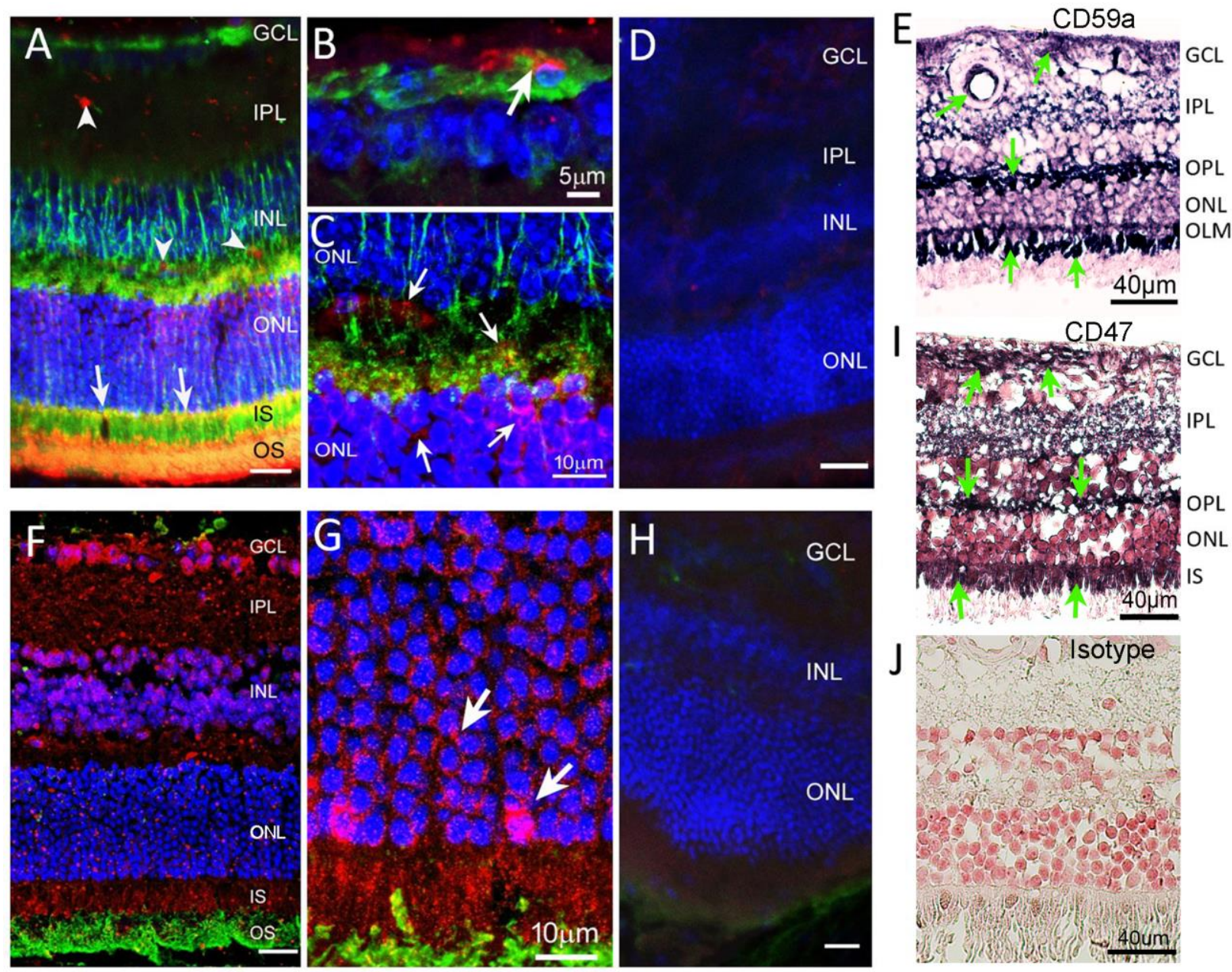

Figure 5. CD59a and CD47 expression in mouse and human retina. (A-C) Retinal sections from 3 months old mice were stained for arrestin C (Green) and CD59a (red) and imaged by confocal microscopy. (A) Low-magnification image showing CD59a in different retinal layers. Arrows indicate CD59a expression in the area of outer limiting membrane; arrowheads indicate discrete CD59a expression in retinal inner layers. (B) High magnification image showing CD59a expression in ganglion cell/nerve fibre layer (arrow). (C) High magnification image showing CD59a expression in outer plexiform - outer nuclear junction. Arrows indicating CD59a ${ }^{+}$staining. (D) Isotype control with DAPI staining. Scale bar in A\&D $=20 \mu \mathrm{m}$. (E) Immunohistochemistry of CD59a in human retina. Arrows indicating CD59a positive staining. (F) Retinal sections from 3 months old mice were stained for rhodopsin (green) and CD47 (red) and imaged by confocal microscopy. Scale bar $=20 \mu \mathrm{m}$. (G) High magnification image showing CD47 expression in photoreceptor nuclear layers (arrows). (H) Isotype control staining. (I) Immunohistochemistry of CD47 in human retina. Arrows indicating CD47 positive staining (J) Isotype control. GCL ganglion cell layer; INL - inner nuclear layer; ONL - outer nuclear layer; OLM - outer limiting membrane; IS - inner segments; OS - outer segments. Representative images from 5 mice and 2 human donors. 

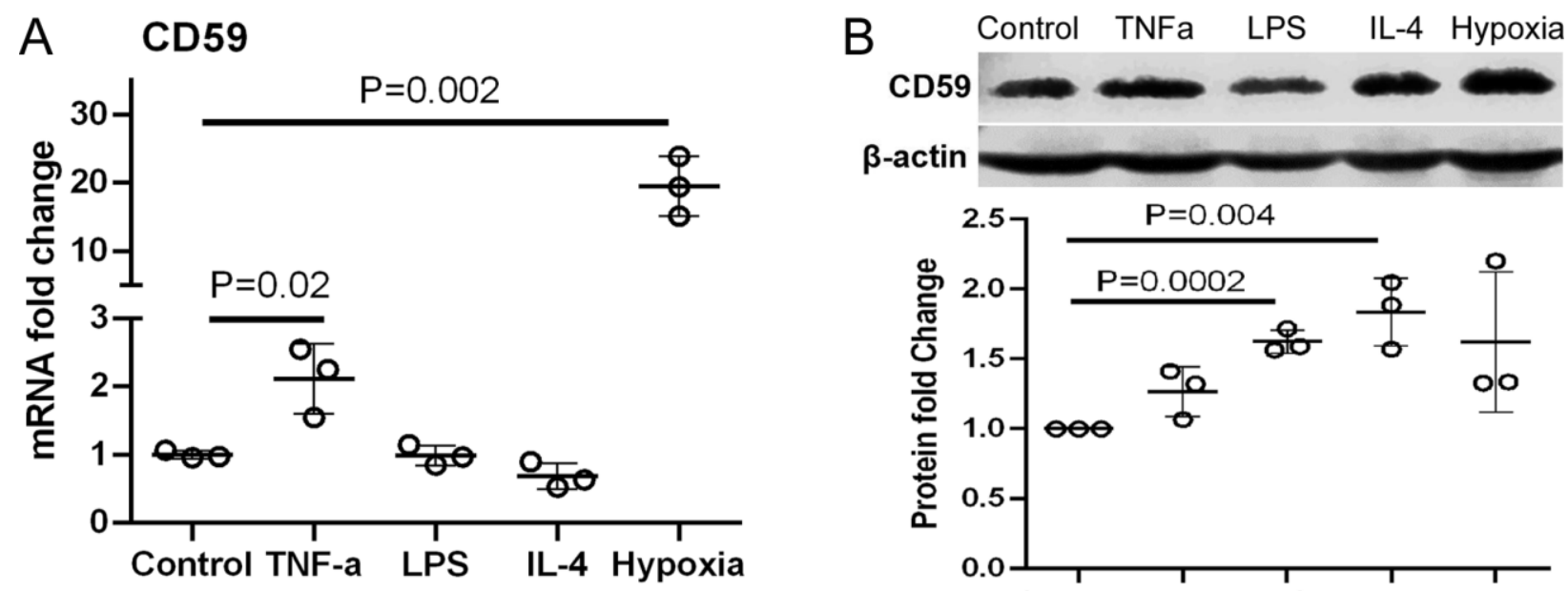

C $\quad$ CD47
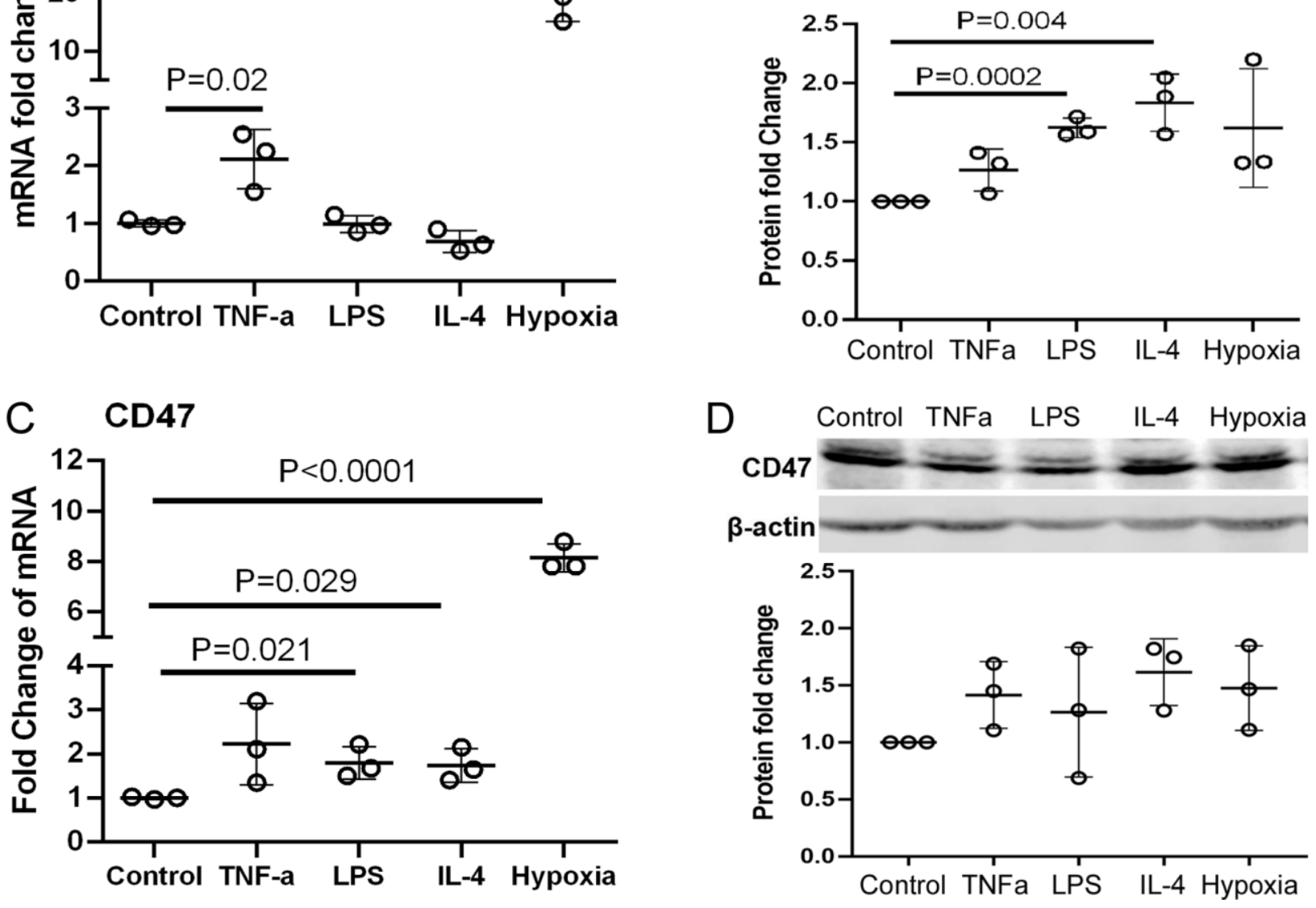

Figure 6. CD59a and CD47 expression in $661 \mathrm{~W}$ cell following inflammatory and hypoxia treatment. $661 \mathrm{~W}$ cells were treated with TNF $\alpha(20 \mathrm{ng} / \mathrm{ml})$, LPS $(100 \mathrm{ng} / \mathrm{ml})$, IL-4 $(20 \mathrm{ng} / \mathrm{ml})$ or hypoxia $\left(5 \% \mathrm{O}_{2}\right)$ for $18 \mathrm{~h}$. Total RNA and protein were extracted and processed for real-time RTPCR (A, C) or Western Blot (B, D). (A) CD59a mRNA expression in different treatment groups; (B) Representative image of Western Blot for CD59a and dot-plot of protein fold changes in different groups from three independent experiments. (C) CD47 mRNA expression in different treatment groups. (D) Representative image of Western Blot for CD47 and dot-plot of protein fold changes in different groups from three independent experiments. Mean $\pm \mathrm{SD}, \mathrm{N}=3$. Unpaired Student's t-test. 

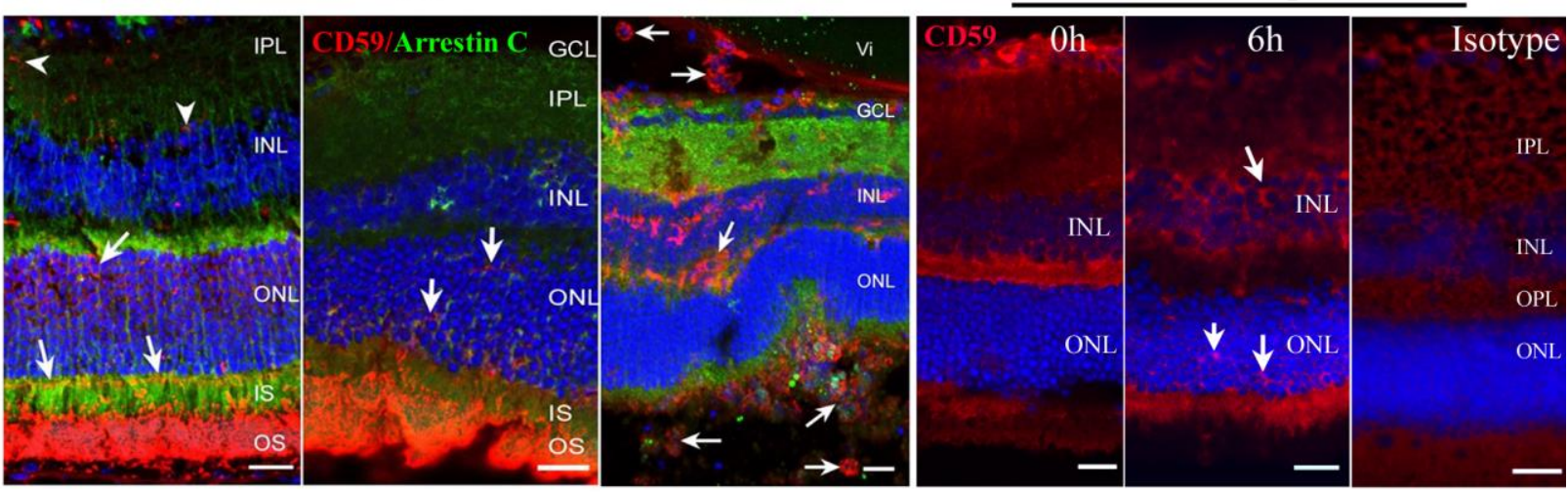

B Control

$\mathrm{RD}$

EAU

Retinal explant
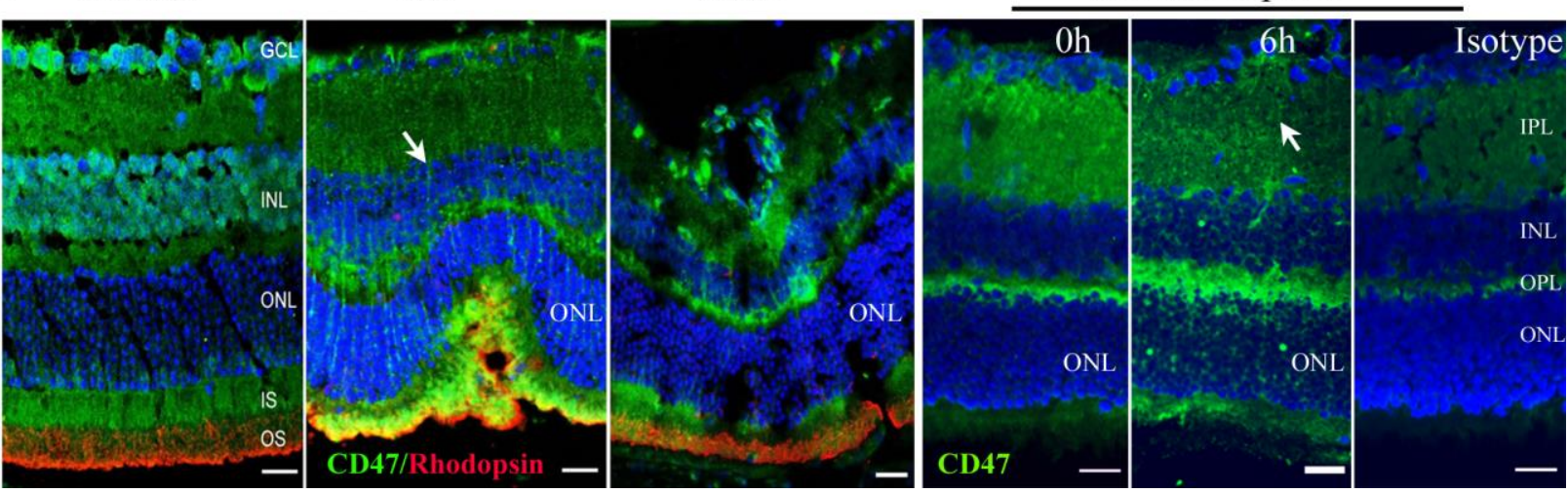

Figure 7. The expression of CD59a and CD47 in the detached retina, inflamed retina and cultured retinal explants. (A) Retinal sections from normal mice and mice with one-week retinal detachment (RD) or day 25 experimental autoimmune uveoretinitis (EAU) were stained for arrestin C (green), CD59a (red) and DAPI. Cryosections from fresh isolated retinal explants from normal mice or retinal explants cultured for 6h were stained for CD59a (red) and DAPI. (B) Retinal sections from normal mouse, mice with one-week RD or day 25 EAU were stained for rhodopsin (red), CD47 (green) and DAPI. Cryosections from fresh isolated retinal explants from normal mice or retinal explants cultured for $6 \mathrm{~h}$ were stained for CD47 (green) and DAPI. All samples were imaged by confocal microscopy. Vi - vitreous; GCL - ganglion cell layer; IPL inner plexiform layer; INL - inner nuclear layer; ONL - outer nuclear layer; IS - inner segments; $\mathrm{OS}$ - outer segments. Scale bars $=20 \mu \mathrm{m}$. Representative images from 5 mice in each group.

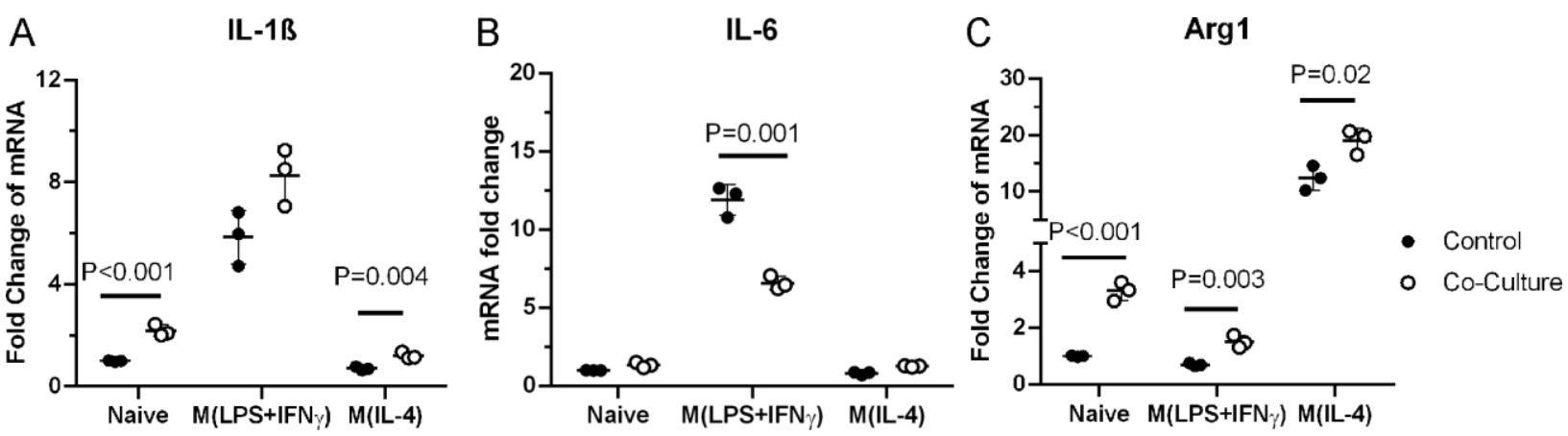

Figure 8. The expression of IL-1b, IL-6 and arginase-1 mRNA in BV2 cells co-cultured with 661W. Control BV2 cells (Naïve), or LPS (100 ng/ml) + IFN $\gamma(20 \mathrm{ng} / \mathrm{ml})$-treated or IL-4 (20 $\mathrm{ng} / \mathrm{ml})$-treated BV2 cells were co-cultured with $661 \mathrm{~W}$ cells $(\mathrm{BV} 2: 661 \mathrm{~W}=5: 1)$ for $24 \mathrm{~h}$. BV2 cells were then isolated using CD11 b ${ }^{+}$MACS kit. The mRNA expression of IL-1b (A), IL-6 (B) and 
Arginase-1 (C) was examined by real-time RT-PCR. M(LPS+IFN $\gamma)-$ BV2 cells treated with LPS+IFN $\gamma ;$ M(IL-4) - BV2 cells treated with IL-4. Mean \pm SD, $N=3$. Unpaired Student t test.

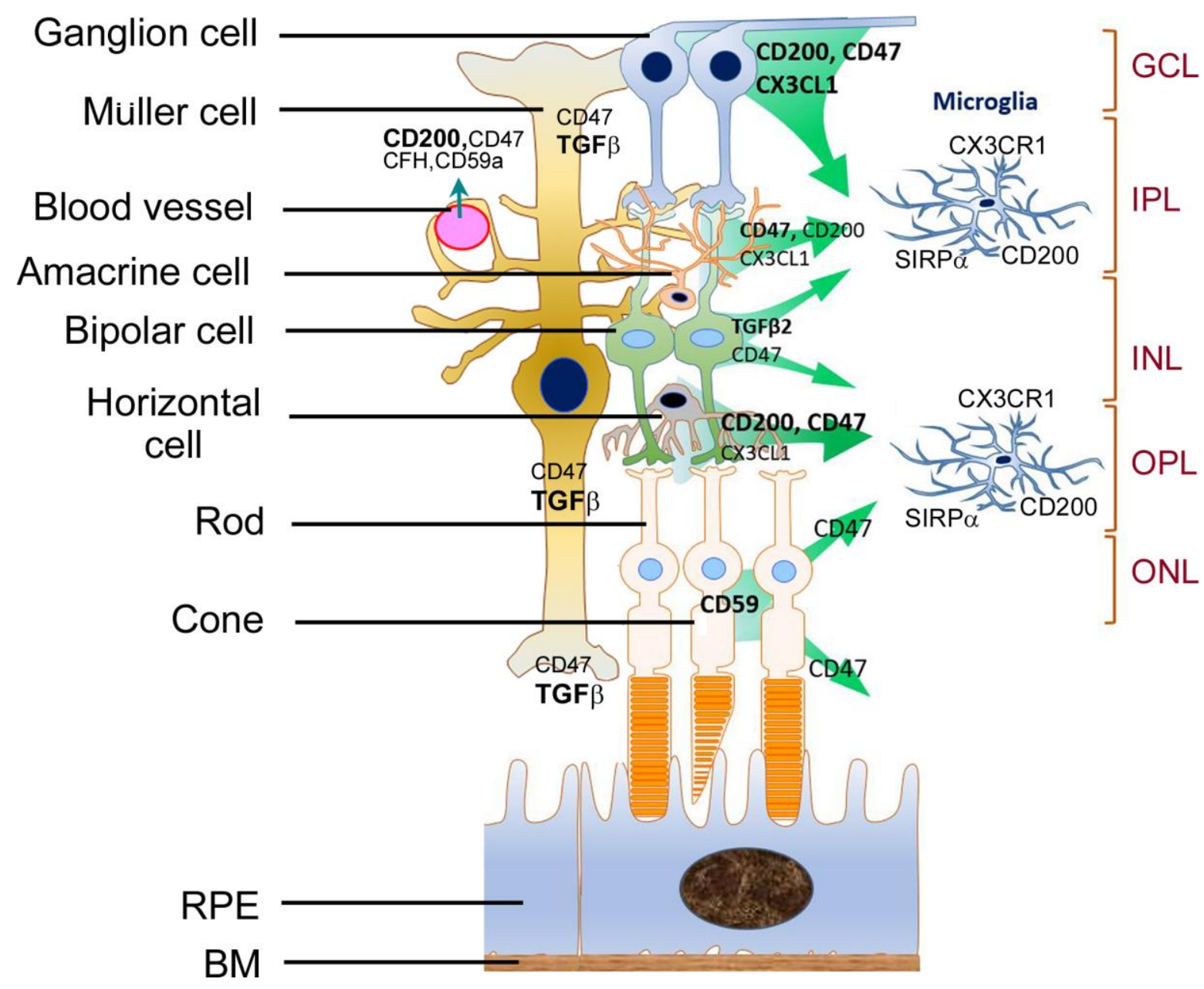

Figure 9. The potential role of neurons in regulating retinal immune activation. In the inner retina (from GCL to IPL), ganglion cells express high levels of CD200, CD47 and CX3CL1, followed by amacrine cells. Vascular endothelial cells express CD200 and CD47. They also express complement factor $\mathrm{H}(\mathrm{CFH})$ and $\mathrm{CD} 59 \mathrm{a}$. In the outer retina (from INL to ONL), horizontal cells express high levels of CD200 and CD47; whereas, bipolar cells express only low-levels of TGF $\beta 2$ and CD47. Photoreceptors, on the other hand, express high-levels of CD59 and CD47. Immune activation, including microglial and complement activation, in the inner retina may be regulated predominately by ganglion cells and vascular endothelial cells with the support from amacrine cells. Whereas, immune activation in the outer retina may be regulated by horizontal cell and photoreceptors. Müller cells, which span throughout the neuroretina, express TGF $\beta 2$ and CD47 and may participate in the regulation of all types of retinal immune activation. Bold large font indicates high levels of expression. GCL - ganglion cell layer; IPL - inner plexiform layer; INL - inner nuclear layer; OPL - outer plexiform layer; ONL - outer nuclear layer; RPE - retinal pigment epithelium; BM - Bruch's membrane. 